Ensayo Original / Original Essay

\title{
Las Tecnologías de Información y Comunicación: Una Gestión Educativa desde la Plataforma Moodle
}

\author{
Autora: Nazare del Valle Marín Parra \\ Universidad Pedagógica Experimental Libertador, UPEL \\ nymangelic2028@gmail.com \\ Puerto Cabello, Venezuela \\ https://orcid.org/0000-0003-4397-5249
}

Resumen

El estudio tiene como fin describir el uso de la plataforma Moodle como herramienta de aprendizaje a distancia (e-learning) que permite ayudar a los educadores a gestionar cursos educativos, el cual apoyan los aprendizajes de los estudiantes a quien está dirigido. En este sentido, se realizó la investigación se encuentra bajo un enfoque analítico cualitativo a través del cual permite que el fenómeno objeto de estudio, sea analizado y a su vez explicada para su posterior comprensión cuyo propósito fundamenta la investigación, en cuanto al aspecto teórico se respaldó por la Teoría Conectivista cuyo aporte se visualiza a través del aprendizaje significativo, el constructivismo. Las conclusiones aproximadas arrojaron que Moodle se considera un instrumento de aprendizaje a distancia (e-learning) que permite asistir a los profesores a establecer enseñanza de calidad en línea, el cual apoya los aprendizajes de los estudiantes a quien está dirigido. La herramienta Moodle permite a los estudiantes la realización de sus aprendizajes y pueden ayudarse por medio de la experiencia educativa en muchas formas.

Palabras clave: aprendizaje en línea; tecnología educacional; gestión educacional; sistema de información educativa; técnica didáctica. 


\title{
Technologies of Information and Communication: An Educational Management from the Moodle Platform
}

\begin{abstract}
The purpose of the study is to describe the use of the Moodle platform as a tool for distance learning (e-learning) that allows educators to help manage educational courses, which support the learning of the students to whom it is addressed. In this sense, the research was conducted under a qualitative analytical approach through which allows the phenomenon under study, is analyzed and explained in turn for further understanding whose purpose is the basis of the research, as the theoretical aspect was supported for the Conectivist Theory whose contribution is visualized through meaningful learning, constructivism. The approximate conclusions showed that Moodle is considered an instrument of distance learning (e-learning) that allows teachers to assist in establishing quality online teaching, which supports the learning of the students to whom it is addressed. The Moodle tool allows students to carry out their learning and can be helped through the educational experience in many ways.
\end{abstract}

Keywords: online learning; educational technology; educational management; educational information system; didactic technique. 


\section{Introducción}

Las Tecnologías de la Información y la Comunicación (TIC) generan un gran impulso desde su aparición e incorporación al mundo de hoy, este posee un gran impacto a todos los campos de la sociedad. En materia educativa no es la excepción, por tal razón se inicia la propuesta que se orienta a su uso para difundir elementos para el desarrollo intelectual de los participantes en estudio y al mismo tiempo les permitirá contar con un recurso que les ayudará a conocer el uso de la plataforma Moodle como una herramienta en el medio de enseñanza virtual inteligente.

Es así, el uso de las herramientas tecnológicas ofrece muchas ventajas en la formación de los estudios especiales educativos en cuanto podemos referirnos como: al aumento explicativo, producción de entornos flexibles para el aprendizaje andragógico, favorece tanto el aprendizaje autónomo como el aprendizaje colaborativo a través de una variedad de estrategias.

Como expone Martínez (2016): los programas de formación docente de las Tecnologías de Aprendizaje y Comunicación, "requieren de una estructura organizativa, la cual debe estar bajo la figura de una coordinación que se encargue de organizar todo lo relativo a los desarrollos de estos proyectos" (pág. 90).

Asimismo, cuando se encuentran grandes avances tecnológicos en información y comunicación, el aprovechamiento de estos significa un gran paso para mejorar el proceso educativo; en tal sentido nos queda hacer uso de todas estas herramientas tecnológicas y creativas para tratar en lo posible que los usuarios internalicen el contenido de dicha aplicación.

En razón de lo antes planteado la intención del presente ensayo es describir la aplicabilidad de las Tecnologías de Información y Comunicación (TIC) dentro de la gestión educativa desde la plataforma Moodle en la que se visiona las funciones que briden y proporcionen asistencia de acuerdo a la necesidad del docente y los modelos educativos que el instituto maneje, 
incorporando el uso de las tecnologías al desarrollo y aprendizaje, a través se superen obstáculos como el tiempo y espacio, brindando varios mecanismos que el docentes y sus estudiantes la empleen para desarrollar los planes de estudios.

La evolución habitual de cambio, admite instrumentos como la mensajería directa e inmediata, vínculos, links, foros, talleres, documentos, cuestionarios, diálogos entre otras, de formas asincrónicas y viceversas de estructura. Con la herramienta Moodle, el docente no precisa poseer instrucción técnica determinada para establecer comunidades educativas en la Web, simplemente hay que brindar actividad docente. Igualmente, consienten otras formas de implantar la práctica en el aula desarrollando la investigación a distancia y así descubrir el uso adecuado de las plataformas inteligentes a través de la web y fuera de ella, de la misma forma de la situación educativa está centralizada en los participantes que trascienden el progreso del propósito crítico y creativo mediante el trabajo en colectivo.

\section{Desarrollo}

Las Tecnologías de la Información y la Comunicación (TIC) generan una mejora desde su aparición e incorporación al mundo en esta época, este marco en completo a todos los campos de nuestra sociedad. En materia educativa no es excepción, por tal razón se busca de describir la gestión educativa desde la plataforma Moodle, como medio de enseñanza virtual inteligente que se orienta como medio de divulgar elementos para el desarrollo intelectual de los participantes en estudio y al mismo tiempo les permitirá contar con un recurso que les ayudará a conocer esta técnica de aprendizaje a distancia en algunos casos citados Ambientes de Aprendizaje Virtual o Educación en Línea.

En otras palabras, es necesario que se describa a partir de un análisis crítico que la Educación a distancia es un curso de enseñanza que debe estar 
centrado para el estudiante, a fin de ajustarse a los cambios que se están generando y garantizar de esta forma la adecuación del currículo a las necesidades, intereses y aspiraciones del estudiante y desde esta realidad promover la construcción permanente del conocimiento.

Desde ese marco de acción, el colectivo en las últimas épocas, demanda incorporar el uso de herramientas que se adapten a los recientes cambios que se están planteando en el mundo globalizado de hoy en todas las áreas del conocimiento. Es por tal motivo, que la labor que ha realizado el hombre en pro de la ciencia informática, se ha convertido en la producción de las Tecnologías de la Información y la Comunicación (TIC), las cuales se definen por ser un conjunto de dispositivos tienen como finalidad la mejora de la calidad de vida de los seres humanos.

Asimismo, la Reunión Regional de Consulta de América Latina y el Caribe de la Conferencia Mundial sobre Ciencia de la UNESCO (1999): sostiene en sus conclusiones que "el desarrollo vertiginoso del entorno virtual obliga a establecer incentivos para estimular la adaptación a las nuevas formas de comunicación e interacción a fin de minimizar los desajustes de capacidades mediante la formación de usuarios claves" (pág. 8).

Es por ello que, se considera que las nuevas formas de comunicación e interacción son un incentivo importante, en el cual es necesario que los interesados participen en esa formación de los entornos virtuales y así conocer a través de estas plataformas las dinámicas que se emplean tales como: clase magistrales, dinámicas en grupos, seminarios, tutorías, asesorías, conferencias entre otros, es por todo esto que resulta necesario implantar las TIC a través de la plataforma virtual Moodle.

Desde esa perspectiva, la creación de estos nuevos ambientes de aprendizajes simultáneamente con el uso de las nuevas e innovadoras herramientas tecnológicas, permiten por una parte, variadas formas de introducir la práctica en el aula desarrollando la investigación a distancia y así 
experimentar el uso adecuado de las plataformas inteligentes a través de la web y fuera de ella, asimismo la producción de un sistema educativo absorto en los participantes donde se promueve el avance la corriente crítica y creativa a través del esfuerzo en equipo.

Ante lo planteado, el uso de las herramientas tecnológicas brinda muchas ventajas en la formación de los estudios especiales educativos entre ellas pueden referirse el desarrollo de propuestas explicativas, creación con ambientes flexibles para la enseñanza andragógica, favorece al aprendizaje independiente como el aprendizaje colaborativo a través de una variedad de estrategias.

Desde esa configuración, se continúan con la panorámica del escenario a nivel mundial, en el que se evidencia que se han generados cambios y transformaciones que requieren la incorporación de instrumentos para intensificar el trabajo en cualquier área igualmente de un sistema de información interconectado para terminar con los impedimentos que están en el medio comunicacional.

Al respecto, Fandos, Jiménez y Pío (2002), refiere que:

La utilización de las redes ha demostrado su capacidad al ser utilizadas en aspectos como la transmisión de la información, de conocimiento y en la comunicación además de afianzar la idea de incorporar estos recursos al proceso de formación. Se trata de un nuevo el paradigma formativo mediante el abordaje de aspectos como comunicación didáctica mediada a través de estrategias de carácter virtual e interactivo (pág. 28).

Por lo tanto, los sistemas de información son un incentivo que debe ser manejado por los usuarios, para que se logre ampliar el conocimiento a través de las diversas transmisiones de información. Pareciendo que estos recursos permiten ser abordados mediante estrategias que se manejen a nivel virtual e interactivo, logrando con ellos un proceso de formación que consolidara la comunicación. 
Lo descrito anteriormente se evidencia en Venezuela, en el que se presenta el auge de utilizar las plataformas virtuales y como parte de ellas existen los diferentes tipos que manejan de una forma limitada la información como prensas, libros entre otros de este tipo. Ahora bien, a través de las plataformas inteligentes como lo es Moodle y con la ayuda de las redes de comunicación Web, se manifiesta un gran potencial para el manejo de la información.

En este momento existen muchas plataformas educativas libres como privadas que proporcionan diferentes instrumentos para la administración pedagógica, como Moodle, que permite respaldar el manejo colectivo como, además al mismo tiempo admite conferencia personal entre los alumnos. Tal como lo plantea, Salinas (2004), quien señala que: "Moodle es una plataforma de aprendizaje a distancia (e-learning) basada en software libre que cuenta con una grande y creciente base de usuarios" (pág. 13); es decir, es un ambiente tácito trazado para asistir a los docentes a establecer un desarrollo óptimo en línea y se apoyan los aprendizajes de los estudiantes a quién está dirigido.

En ese orden de ideas, en este ámbito intervienen estas tecnologías como estrategias y técnica formativo innovador para el curso de enseñanza y desarrollo. De manera que, constituyen elementos facilitadores, que promueven el desarrollo de aptitudes intelectuales, la adquisición de habilidades y destrezas tanto en el estudiante como en el docente y su posterior fortalecimiento.

Con base a lo expuesto, se requiere que el estudiante tenga la posibilidad de establecer encuentros bajo mecanismos de comunicación sincrónica y asincrónica soportada por las TIC, también de poder formar grupos de aprendizaje bajo un mismo objetivo e interés colectivo. Es por ello que, la Teoría Conectivista o Conectivismo es una teoría del aprendizaje promovido por Siemens (2005): "Donde la llamada la teoría del aprendizaje 
para la era digital, se trata de explicar el aprendizaje complejo en un mundo social digital en rápida evolución" (párr. 1).

Su aporte se visualiza a través del aprendizaje significativo, el constructivismo en el que se evidencia la necesidad de aprender y construir su propio aprendizaje, a través del Moodle como una herramienta que ofrece apoyo para complementar el aprendizaje y el manejo efectivo de las tecnologías.

Ahora bien, la filosofía trazada por Moodle circunscribe un acercamiento constructivo apoyado en el edificante impulso social de la enseñanza, acentuando que los alumnos pueden ayudar a la práctica educativa en diversas formas. Habiendo dicho esto, Moodle se adecua de forma flexible y así permitir un extenso grado de modos de instrucción.

Se considera entonces que, la creación de estos nuevos ambientes de aprendizajes simultáneamente con el uso de las nuevas e innovadoras herramientas tecnológicas, permiten por una parte, otras formas de fundar la práctica en el aula desarrollando la investigación a distancia y así percibir el uso adecuado de las plataformas inteligentes a través de la web y fuera de ella, asimismo de la creación de una ambiente educativo dinámico en los participantes que promueven el progreso de la tendencia crítica y creativa por medio de la interacción grupal.

\section{Conclusiones}

En respuesta a propósito de este ensayo, se logra a partir de una visión epistémica relacionadas a las tecnologías de Información y Comunicación (TIC) en el que se puede gestionar desde una plataforma educativa virtual Moodle, el cual se configura como un entorno informático con varias herramientas concentradas y optimadas hacia fines educativos. Por ello, los entornos virtuales permiten acceder a la realización y ordenamiento de los recursos completos para internet, que sean precisos los conocimientos, 
dejando claro que Moodle es una plataforma de aprendizaje a distancia (elearning) que permite asistir a los profesores a establecer instrumentos de eficacia en línea y donde esta apoyan los aprendizajes de los alumnos a quién está dirigido.

Asimismo, estos instrumentos son de mucha utilidad a nivel educativo, ya que aprueban a los docentes la administración de cursos virtuales para sus estudiantes (formación a distancia o educación en línea), la utilización de un espacio en línea que se apoye con la presencial.

De igual manera, la herramienta Moodle permite renovar en los alumnos, un método de enseñanza para la adquisición de aprendizajes y a su vez puedan ayudarse por medio de una experiencia educativa en muchas formas, destacando que los alumnos (y no solo los profesores) contribuyen a la práctica educativa de diferente manera.

Aunado a ello se debe entender que mediante la construcción de estos nuevos ambientes de aprendizajes juntamente con el uso de las nuevas e innovadoras herramientas tecnológicas, permitirá de una forma de constituir la práctica en el aula ampliando la investigación a distancia y así apreciar el uso adecuado de las plataformas inteligentes a través de la web.

\section{Referencias}

Martínez, O. (2016). Programa de Formación Docente de las Tecnologías del Aprendizaje y el Conocimiento (TAC) En la Universidad Pedagógica Experimental Libertador Núcleo Barinas (Venezuela). Revista Scientific, 1(1), 90-114. Recuperado de:

https://doi.org/10.29394/scientific.issn.2542-2987.2016.1.1.6.90-114

Fandos, M., Jiménez, J., \& Pio, A. (2002). Estrategias didácticas en el uso de las tecnologías de la información y la comunicación. Acción Pedagógica, 11(1), 28-39, ISSN-e: 1315-401X. Recuperado de:

https://dialnet.unirioja.es/servlet/articulo?codigo=2973066 
Salinas, J. (2004). Innovación docente y uso de las TIC en la enseñanza universitaria. Revista de Universidad y Sociedad del Conocimiento RUSC, 1(1), 1-16, ISSN: 1698-580X. Recuperado de:

http://dx.doi.org/10.7238/rusc.v1i1.228

Siemens, G. (2005). Connectivism: A Learning Theory for the Digital Age. International Journal of Instructional Technology \& Distance Learning, 2(1), ISSN: 1550-6908. Recuperado de:

http://www.itdl.org/journal/jan 05/article01.htm

UNESCO (1999). Reunión regional de consulta de América Latina y el Caribe de la Conferencia mundial sobre la ciencia. La ciencia para el siglo XXI: una nueva visión y un marco de acción. Santo Domingo, República Dominicana: United Nations Educational, Scientific and Cultural Organization. Recuperado de:

http://www.unesco.org/science/wcs/meetings/lac santo domingo s 9 9.htm 


\section{Nazare del Valle Marín Parra \\ e-mail: nymangelic2028@gmail.com}

Nacida en Puerto Cabello, Estado Carabobo,

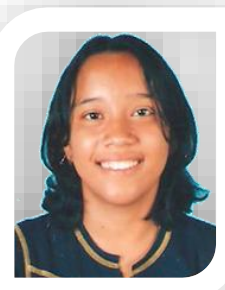

Venezuela. Ingeniero en Informática de la Universidad Alejandro de Humboldt; T.S.U. en Informática del Instituto Universitario de Tecnología Juan Pablo Pérez Alfonso; Impartió docencia en el área de informática en el Instituto Universitario de Tecnología Juan Pablo Pérez Alfonso, Universidad José Antonio Páez y Misión Sucre; Diplomado en Docencia Universitaria en el Instituto Universitario de Tecnología Juan Pablo Pérez Alfonso; Me desempeño como Analista de Sistema en el Instituto Universitario de Tecnología de Puerto Cabello IUTPC; Realizo proyectos de Sistemas de Información a través de la Empresa Venezolana de Electrónica y Programación Industrial EVEMPI, C.A. Cuenta con 10 años de experiencia en el área docente en el Sistema Universitario y 13 años de experiencia a nivel Universitario en las áreas de Tecnología de la Información y Comunicación, Metodología de la Investigación, entre otras, en diferentes universidades e institutos universitario (IUTPC, IUTEPAL, UJAP, Misión Sucre). Colaboradora y participante en distintos eventos culturales, pedagógicos y académicos. He realizado diferentes cursos, talleres, jornadas y seminarios en el área de Tecnología, Educación, Investigación y distintos saberes del conocimiento.

El contenido de este manuscrito se difunde bajo una Licencia de Creative Commons ReconocimientoNoComercial-Compartirlgual 4.0 Internacional 